\title{
Degradation of histamine by the halotolerant Staphylococcus carnosus FS19 isolate obtained from fish sauce
}

\begin{abstract}
Histamine is found in many fermented food products and may have detrimental effects on the health of its consumers. Histamine and other amines are degraded by the oxidative deamination activity of certain microorganisms. In this study, the growth characteristics and histamine-degrading activity of a Staphylococcus carnosus FS19 isolate derived from fish sauce were investigated. This bacterium exhibits optimal growth at $35{ }^{\circ} \mathrm{C}, \mathrm{pH} 8$ and $9 \%$ sodium chloride when cultivated in tryptic soy broth. The histamine-degrading activity of the S. carnosus FS19 isolate was optimised at $40{ }^{\circ} \mathrm{C}$ and pH 6 in $9 \%$ buffered sodium chloride. When added to fish sauce samples, this bacterium exhibits remarkable histamine-degrading activity. The histamine concentration was reduced by approximately $15.1 \%$ and $13.8 \%$ in the fish sauce samples that contained $18 \%$ and $21 \%$ salt, respectively. However, no histamine degradation was observed in samples with a salt content greater than $21 \%$. In addition, a slight degradation of other amines, including putrescine and cadaverine, was also observed in some of the samples. In contrast, tyramine degradation did not occur in any of the samples. Therefore, S. carnosus FS19 is a culture that could potentially reduce the histamine content of fermented fish products.
\end{abstract}

Keyword: Histamine; Biogenic amines; Histamine degradation; Staphylococcus carnosus; Fish sauce 